\title{
Sleep Disturbances in Myotonic Dystrophy Type 2
}

\author{
Paul Shepard Erek M. Lam Erik K. St. Louis Jacob Dominik
}

Mayo Center for Sleep Medicine, Division of Pulmonary and Critical Care Medicine, Departments of Medicine and Neurology, Mayo Clinic and Foundation, Rochester, Minn., USA

\section{Key Words}

Myotonic dystrophy type $2 \cdot \mathrm{DM} 2 \cdot$ Excessive daytime

sleepiness $\cdot$ EDS $\cdot$ Fatigue $\cdot$ OSA $\cdot$ RLS

\begin{abstract}
Sleep disorders in myotonic dystrophy type 1 (DM1) are common and include sleep-disordered breathing, hypersomnia, and fatigue. Little is known regarding the occurrence of sleep disturbance in myotonic dystrophy type 2 (DM2). We hypothesized that DM2 patients may frequently harbor sleep disorders. We reviewed medical records of all genetically confirmed cases of DM2 seen at our sleep center between 1997 and 2010 for demographic, laboratory, overnight oximetry, and polysomnography (PSG) data. Eight patients ( 5 women, 3 men) with DM2 were identified. Excessive daytime sleepiness was seen in 6 patients (75\%), insomnia in $5(62.5 \%)$, and excessive fatigue in $4(50 \%)$. Obstructive sleep apnea was diagnosed in 3 of 5 patients (60\%) studied with PSG. Respiratory muscle weakness was present in all 6 patients (100\%) who received pulmonary function testing. Four of $8(50 \%)$ met criteria for diagnosis of restless legs syndrome. The clinical spectrum of DM2 may include a wide range of sleep disturbances. Although respiratory muscle weakness was frequent, sustained sleep-related hypoxia suggestive of hypoventilation was not seen in our patients. Further prospective studies are needed to examine the frequency and scope of sleep disturbances in DM2.
\end{abstract}

Copyright $\odot 2012$ S. Karger AG, Basel

\section{Introduction}

Myotonic dystrophies are dominantly inherited, progressive, multisystem disorders characterized by neuromuscular weakness, myotonia, early cataracts, endocrine abnormalities, and cardiac arrhythmias $[1,2]$. There are 2 heterogeneous, but clinically and genetically distinct forms of myotonic dystrophy: type 1 (DM1) and type 2 (DM2). A high degree of clinical overlap exists between these two entities [1-3].

DM1 results from a trinucleotide CTG unstable repeat expansion on chromosome 19q13.3 [1,3,4]. After the identification of the CTG trinucleotide repeat expansion in the DMPK (myotonic dystrophy protein kinase) gene, reports began to emerge of phenotypically similar patients not harboring this mutation $[2,5]$. Distinctive clinical features in this subgroup included the propensity for less severe, proximal weakness, and lack of prominent muscle wasting. This phenotypically distinct myotonic dystrophy, DM2, was found to be due to a CCTG unstable repeat expansion on the ZNF9 (zinc finger protein 9) gene on chromosome 3q21.3 [1, 3, 4]. Unlike DM1, in which increased repeat length causes the phenomenon of genetic anticipation (thereby influencing disease severity and causing earlier symptom development and presentation), in DM2, repeat length does not seem to correlate with severity $[1,2]$.

Clinically, DM2 may be suspected in an adult with proximal lower extremity and finger flexor weakness, pain and stiffness, and electromyographic myotonia [2,

\section{KARGER}

Fax +4161306 1234

E-Mail karger@karger.ch

www.karger.com (c) 2012 S. Karger AG, Basel

0014-3022/12/0686-0377\$38.00/0

Accessible online at:

www.karger.com/ene
Erik K. St. Louis, MD, Assoc. Professor of Neurology

Center for Sleep Medicine, Mayo Clinic and Foundation

200 First Street Southwest

Rochester, MN 55905 (USA)

E-Mail StLouis.Erik@ mayo.edu 
3]. Several key features of DM1 are typically absent in DM2, including facial weakness, ptosis, wasting of temporalis muscles, severe atrophy, and respiratory insufficiency. Nevertheless, differentiation between the two myotonic dystrophies on clinical grounds alone is often difficult, and genetic evaluation is necessary to make a definitive diagnosis [1-4].

Sleep disturbances in DM1 are well described, including frequent hypersomnia, fatigue, and sleep-disordered breathing (SDB) abnormalities characterized by either central or obstructive sleep apnea (OSA) [6-10]. OSA is particularly frequent in DM1 patients, occurring in 30$70 \%$ of patients $[7,10]$. While apnea, hypoventilation, and hypercapnia contribute to sleep disturbances associated with DM1, there is also a primary central disorder of arousal responsible for excessive daytime sleepiness (EDS) intrinsic to DM1 disease pathology $[9,11]$. Shortened latencies on multiple sleep latency tests (MSLT) constitute further evidence for an intrinsic predisposition toward sleepiness in DM1 [8]. Restless legs syndrome (RLS) was recently described in DM1 [12].

Little is known in regards to the occurrence of sleep disturbances in DM2. A recent publication found poor sleep quality and excessive fatigue in DM2; however, in contrast to findings in DM1, EDS was not prevalent in DM2 patients [13]. A better understanding of sleep disturbances in DM2 could improve clinical care of these patients and aid in its distinction from DM1 clinically.

\section{Methods}

We reviewed the medical records of all patients with genetically confirmed DM2 referred to the Mayo Clinic Center for Sleep Medicine, Rochester, Minn., USA, from 1997 to 2010. Demographic data, associated diagnoses, sleep questionnaires, laboratory tests, overnight oximetry, and polysomnographic studies were reviewed. This review was approved by the Mayo Foundation Institutional Review Board.

Inclusion criteria were: documentation of genetically proven DM2 and age $>18$ years at the time of DM2 diagnosis. Exclusion criteria were: lack of genetic confirmation of DM2 diagnosis or age $<18$ years at the time of DM2 diagnosis.

In-lab polysomnography (PSG) and overnight oximetry data were interpreted by a physician trained in sleep medicine. PSG was scored utilizing standard criteria [14]. The Epworth Sleepiness Scale (ESS) questionnaire, a measure of the tendency to fall asleep during 8 different situations, was administered at initial presentation [15]. ESS scores range from 0 to 24 , with a score $>10$ generally considered a reflection of abnormal daytime sleepiness. RLS was defined clinically via standard symptomatology, including an unpleasant sensation of an urge to move the legs, occurring at rest, relieved by movement, and most prominent in the evening hours.

\section{Results}

We identified 8 patients ( 5 women and 3 men) with genetically confirmed DM2 (table 1). Mean age was 62 years (range, 43-82 years), and mean BMI was 29.99 (range, 21.29-42.75). The most common presenting symptom was EDS in 6 patients (75\%).

Mean ESS score was 7.43 (range, 2-14) in the 7 patients who completed the survey. Five patients (62.5\%) reported insomnia and $4(50 \%)$ reported excessive fatigue. Four patients $(50 \%)$ had a history of psychiatric diagnosis of depression.

PSG was completed in 5 patients. Mean sleep efficiency was $74 \%$ (range, 43-96\%), mean apnea hypopnea index (AHI) was 6.4 (range, 1-13). Mild OSA (AHI $\geq 5$ ) was present in 3 patients $(60 \%)$ studied with PSG.

Respiratory muscle weakness was present in all 6 patients $(100 \%)$ evaluated with pulmonary function tests, with a mean maximal inspiratory pressure of $62 \%$ (range, $56-66 \%)$ of the predicted value, and a mean maximal expiratory pressure of $48 \%$ (range, $32-78 \%$ ) of predicted. Despite the observed respiratory muscle weakness, none of the 5 patients who underwent PSG demonstrated sustained sleep-related hypoxia indicative of hypoventilation, as mean oxyhemoglobin saturation was $93 \%$ (range, 91-95\%).

One patient (Patient 5) described lifelong severe hypersomnia, excessive napping, and hypnagogic hallucinations. Diagnostic PSG showed only mild sleep apnea with an AHI of 5. MSLT was performed and was abnormal, with a median sleep latency of $5 \mathrm{~min}$ and mean sleep latency of $8.3 \mathrm{~min}$. A single sleep onset REM period was noted during the fourth nap.

Four of 8 patients (50\%) met criteria for RLS, and 3 others had nonspecific nocturnal leg symptoms. Three of 5 patients with RLS had PSG performed and of these diagnostic mean periodic limb movement index was 19 (range, 8-32) and mean periodic limb movement arousal index was 4 (range, $0-8$ ). Ferritin levels were in the lownormal range in 2 of the RLS patients at 25 and $40 \mu \mathrm{g} / \mathrm{l}$.

\section{Discussion}

The spectrum of sleep disturbances in our DM2 patients included daytime sleepiness and fatigue, insomnia, sleep apnea, and RLS, similar to sleep-related problems previously described in DM1 patients [7-10], suggesting that despite distinct genetic mechanisms, CNS symptomatology may be similar in DM2 and DM1. 
Table 1. Clinical characteristics and PSG results of 8 patients with DM2

\begin{tabular}{|c|c|c|c|c|c|c|c|c|c|c|c|}
\hline $\begin{array}{l}\text { Patient } \\
\text { (sex) }\end{array}$ & $\begin{array}{l}\text { Age } \\
\text { years }\end{array}$ & ESS & Fatigue & Insomnia & Depression & $\begin{array}{l}\text { Respiratory } \\
\text { weakness }^{\mathrm{a}}\end{array}$ & RLS & $\mathrm{AHI}^{\mathrm{b}}$ & $\mathrm{AI}^{\mathrm{b}}$ & $\mathrm{PLMI}^{\mathrm{b}}$ & PLMAI $^{\mathrm{b}}$ \\
\hline $1(\mathrm{M})$ & 75 & 2 & $\mathrm{~N}$ & $\mathrm{~N}$ & $\mathrm{~N}$ & $\mathrm{Y}$ & $\mathrm{Y}$ & $11 / 2$ & $35 / 51$ & $17 / 154$ & $8 / 41$ \\
\hline $2(\mathrm{M})$ & 45 & 13 & $\mathrm{~N}$ & $\mathrm{Y}$ & $\mathrm{Y}$ & $*$ & $\mathrm{~N}$ & $*$ & * & * & * \\
\hline $3(\mathrm{~F})$ & 62 & 6 & $\mathrm{Y}$ & $\mathrm{Y}$ & $\mathrm{Y}$ & $\mathrm{Y}$ & $\mathrm{N}$ & $13 / 0$ & $48 / 34$ & $84 / 33$ & $25 / 7$ \\
\hline $4(\mathrm{~F})$ & 60 & 6 & $\mathrm{Y}$ & $\mathrm{N}$ & $\mathrm{N}$ & $\mathrm{Y}$ & $\mathrm{N}$ & $2 / 3$ & $20 / 23$ & $0 / 0$ & $0 / 0$ \\
\hline $7(\mathrm{M})$ & 43 & 8 & $\mathrm{~N}$ & $\mathrm{Y}$ & $\mathrm{N}$ & $\mathrm{Y}$ & $\mathrm{Y}$ & 1 & 20 & 8 & 4 \\
\hline $8(\mathrm{~F})$ & 82 & 3 & $\mathrm{~N}$ & $\mathrm{Y}$ & $\mathrm{N}$ & $\mathrm{Y}$ & $\mathrm{N}$ & * & * & $*$ & * \\
\hline
\end{tabular}

$\mathrm{Y}=$ present $\mathrm{N}$ = absent; ESS = Epworth Sleepiness Scale; Depression = prior psychiatric diagnosis of depression; RLS = restless legs syndrome; $\mathrm{AHI}=$ apnea hypopnea index; $\mathrm{AI}=$ arousal index; $\mathrm{PLMI}=$ periodic limb movement index; PLMAI = periodic limb movement arousal index.

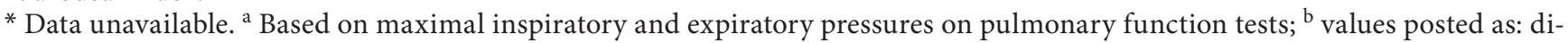
agnostic PSG/PAP titration.

All studies were performed in split-night fashion, except for patient 7 who was a full-night diagnostic PSG. Patients 1, 3, 4, and 5 underwent titration with CPAP, while patient 7 underwent titration with bilevel positive airway pressure in spontaneous mode.

The most common reason for referral of DM2 patients was EDS, yet ESS scores were relatively low in most patients, a finding similar to a recent study reporting poor sleep quality and fatigue in DM2 without prominent EDS [13]. While the ESS is the most widely used tool to assess daytime sleepiness, measurement properties and reliability of the ESS in clinical samples of DM1 patients have previously been brought into question $[7,16]$. A more disease-specific instrument for the assessment of daytime sleepiness in DM2 patients could better evaluate this patient population for hypersomnia. Daytime sleepiness due to primary CNS hypersomnia as described in one of our patients (Patient 5) can be seen in DM2, but it is unclear if this represents a central pathophysiologic mechanism for hypersomnia as has been previously described in DM1.

Interestingly, there were a variety of sleep disturbances in our DM2 cases despite only subtle SDB findings. Sustained sleep-related hypoxia suggestive of sleep-related hypoventilation was absent despite findings of respiratory muscle weakness in each of the patients as assessed by PSG; and OSA, when present, was only of mild severity. However, while DM2 is often considered to manifest a relatively mild degree of weakness relative to DM1, proximal weakness involving respiratory muscles was frequent in our cases. This suggests that pulmonary function testing should be considered in the assessment of DM2 patients, especially those with complaints of sleep disturbance, since respiratory muscle weakness could cause sleep-related hypoventilation that may require bilevel positive airway pressure therapy.

RLS and nonspecific nocturnal leg symptoms were also frequent in our small series of DM2 patients. DM2 patients have previously been noted to be predisposed to prominent myalgias and pain complaints, and a high prevalence of RLS has been described in patients with fibromyalgia and chronic pain $[17,18]$. Restless leg symptoms were also recently reported in DM1 patients [12], but further prospective studies are warranted to better determine the prevalence of restless legs in each DM type.

The clinical spectrum of DM2 appears to include a variety of sleep disturbances, including daytime sleepiness, SDB, RLS, fatigue, and insomnia. Further prospective studies involving a larger patient series with population controls that would be less subject to the inherent limitations of referral and selection biases than our case series are necessary to assess the frequency and nature of sleep disturbances in DM2.

\section{Acknowledgment}

This publication was supported by CTSA Grant Number UL1 TR000135 from the National Center for Advancing Translational Science (NCATS). Its contents are solely the responsibility of the authors ansd do not necessarily represent the official views of the NIH. 


\section{References}

$>1$ Turner C, Hilton-Jones D: The myotonic dystrophies: diagnosis and management. J Neurol Neurosurg Psychiatry 2010;81:358367.

$>2$ Day JW, Ricker K, Jacobsen JF, et al: Myotonic dystrophy type 2: molecular, diagnostic and clinical spectrum. Neurology 2003; 60:657-664.

3 Meola G: Clinical and genetic heterogeneity in myotonic dystrophies. Muscle Nerve 2000;23:1789-1799.

$\checkmark 4$ Liquori CL, Ricker K, Moseley ML, et al: Myotonic dystrophy type 2 caused by a CCTG expansion in intron 1 of ZNF9. Science 2001;293:864-867.

$\checkmark 5$ Ricker K, Koch MC, Lehmann-Horn F, et al: Proximal myotonic myopathy: a new dominant disorder with myotonia, muscle weakness, and cataracts. Neurology 1994;44: 1448-1452.

$\checkmark 6$ Phemister JC, Small JM: Hypersomnia in dystrophia myotonica. J Neurol Neurosurg Psychiatry 1961;24:173-175.

7 Laberge L, Bégin P, Montplaisir J, et al: Sleep complaints in patients with myotonic dystrophy. J Sleep Res 2004;13:95-100.
8 Laberge L, Bégin P, Dauvilliers Y, et al: A polysomnographic study of daytime sleepiness in myotonic dystrophy type 1. J Neurol Neurosurg Psychiatry 2009;80:642-646.

-9 Phillips MF, Steer HM, Soldan JR, et al: Daytime somnolence in myotonic dystrophy. J Neurol 1999;246:275-282.

10 Romigi A, Izzi F, Pisani V, et al: Sleep disorders in adult-onset myotonic dystrophy type 1: a controlled polysomnographic study. Eur J Neurol 2011;18:1139-1145.

11 Ono S, Kurisaki H, Sakuma A, Nagao K: Myotonic dystrophy with alveolar hypoventilation and hypersomnia: a clinicopathological study. J Neurol Sci 1995;128:225-231.

12 Yu H, Laberge L, Jaussent I, et al: Daytime sleepiness and REM sleep characteristics in myotonic dystrophy: a case-control study. Sleep 2011;34:165-170.

13 Tieleman AA, Knoop H, van de Logt AE, et al: Poor sleep quality and fatigue but no excessive daytime sleepiness in myotonic dystrophy type 2. J Neurol Neurosurg Psychiatry 2010;81:963-967.
14 Iber C, Ancoli-Israel S, Chesson A, et al: The AASM manual for scoring of sleep and associated events. Rules, terminology and technical specifications. Westchester, IL: American Academy of Sleep Medicine 2007 pp 45-47.

15 Johns MW: A new method for measuring daytime sleepiness: the Epworth Sleepiness Scale. Sleep 1991;14:540-545.

16 Laberge L, Gagnon C, Jean S, et al: Fatigue and daytime sleepiness rating scales in myotonic dystrophy: a study of reliability. J Neurol Neurosurg Psychiatry 2005;76:14031405.

17 Auvinen S, Suominen T, Hannonen P, et al: Myotonic dystrophy type 2 found in two of sixty-three persons diagnosed as having fibromyalgia. Arthritis Rheum 2008;58:36273631.

18 Viola-Saltzmann M, Watson NF, Bogart A, et al: High prevalence of restless legs syndrome among patients with fibromyalgia: a controlled cross-sectional study. J Clin Sleep Med 2010;15:423-427. 